\section{Mesenchymal cystic hamartoma of the lung: a rare cause of relapsing pneumothorax}

\author{
RJ van Klaveren, H H M Hassing, \\ J M Wiersma-van Tilburg, L K Lacquet, \\ A L Cox
}

\begin{abstract}
A 14 year old boy is described with recurrent spontaneous pneumothoraces due to a mesenchymal cystic hamartoma, a very rare disease with a multicentric nature and a benign course in most patients.
\end{abstract}

(Thorax 1994;49:1175-1176)

We present a very unusual cause of relapsing pneumothorax due to a mesenchymal cystic hamartoma of the lung, a distinct clinicopathological entity first described by Mark in 1986. $^{1}$

University Hospital Nijmegen, Medical Centre Dekkerswald, Groesbeek

$R$ J van Klaveren

A L Cox

Department of

Pulmonary Diseases,

St Maartensgasthuis,

Venlo

H H M Hassing

Department of Pathology

J M Wiersma-van

Tilburg

Department of Thoracic and Cardiac

Surgery,

L K Lacquet

University Hospital

Nijmegen, The

Netherlands

Reprint requests to:

Dr R J van Klaveren,

Department of Pneumology,

Uepartment of Pne

Herestraat 49,3000 Leuven, Belgium.

Received 18 May 1993

Returned to authors

6 July 1993

Revised version received

9 December 1993

Accepted for publication

Accepted for pub

\section{Case report}

A 14 year old Turkish boy, born in the Netherlands, was admitted for a left spontaneous pneumothorax once in August and twice in September 1990. They were unsuccessfully treated with suction, and later by pleurodesis with talc, tetracycline, and blood. The patient had one small haemoptysis, but denied dyspnoea, fever, night sweats, or chills. His past was unremarkable with no history of tuberculosis, recurrent respiratory infections, or smoking. Physical examination revealed dullness and increased breath sounds at the left base. Routine biochemical studies, haematology and urine analysis were normal. The chest radiograph and computed tomographic scan showed a partial left pneumothorax and an atelectatic left lower lobe. Bronchoscopy revealed normal airways. In October 1990 a thoracotomy was performed for persistent pneumothorax. The left lower lobe was collapsed and multiple adhesions and a thickened pleura were found, but no bullae or blebs. In the lateral part of the lower lobe a bloody craterlike defect was found from which a biopsy sample was taken. The initial diagnosis of this biopsy material was pulmonary blastoma. For complete surgical removal of the tumour the patient was referred to the University Hospital in Nijmegen. A lobectomy was performed and a yellow-brown tumour of about $5 \mathrm{~cm}$ in diameter with a superficial defect was found. Pathological examination showed glassy cystic tumours in several places and some fibrosis. Microscopy showed epithelial structures of varying size in which the epithelium was flat cuboid to low cylindrical. The nuclei were sometimes slightly atypical, the surrounding stroma being cellular and consisting of sinuous bundles of long elongated cells. The finding of several nodules containing cystic spaces lined by cuboidal epithelium and a band-like layer of cells (cambium layer) composed of mesenchymal-looking cells with dark nuclei and scanty cytoplasm is in keeping with a diagnosis of mesenchymal cystic hamartoma (figure).

Two years after thoracotomy the patient is well without evidence of recurrence or metastasis.

\section{Discussion}

This case report illustrates that mesenchymal cystic hamartoma of the lung enters into the differential diagnosis for a patient with pneumothorax or haemoptysis and nodules or cysts on a chest radiograph. By strict definition the

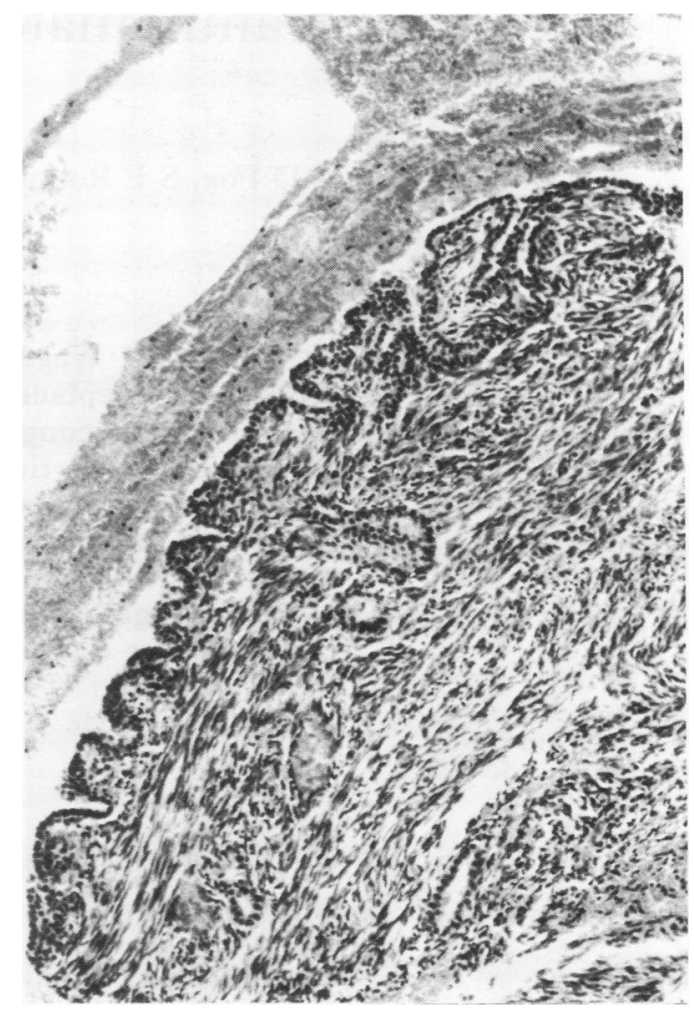

Cyst lined by cuboidal epithelium and stroma composed of mesenchymal-looking cells with dark nuclei and scanty cytoplasm. Stain: haematoxylin and eosin, original magnification $\times 150$ reduced to $65 \%$ in origination. 
disordered growth of both endodermal (respiratory epithelium) and mesodermal (vascular) elements satisfies criteria for the pathological diagnosis of a hamartoma. ${ }^{2}$ Primitive mesenchyme is fetal tissue. By this definition and according to the hypothesis of Mark, ${ }^{1}$ mesenchymal cystic hamartoma of the lung is hamartomatous and congenital. Only seven cases have been reported to date. The nodules and cysts increase very slowly over a period of many years and, although the disease may be detected in infancy, ${ }^{3}$ three or four decades usually elapse before it comes to clinical attention. ${ }^{14}$ Neither the epithelial nor the mesenchymal cells have malignant features, but malignant degeneration has been described. ${ }^{5-7}$ Nodules and cysts represent different stages of the disease. ${ }^{1}$ The nodules arise from mesenchyme proliferation in the interstitium. When the nodules reach $1 \mathrm{~cm}$ in diameter bronchiolectasis occurs and small cysts form, lined with normal or metaplastic bronchiolar epithelium. Bleeding from systemic arteries into the cysts causes haemoptysis, and rupture of subpleural cysts causes pneumothorax or haemothorax. New nodules continue to appear when the cysts have already developed. Surgery is needed for diagnosis and for treatment of a pneumothorax or haemothorax. The possible benefit of resecting nodules and cysts to preclude malignant transformation must be weighed against the multicentric nature and benign course in most patients.

We thank M Koss, Chairman, Department of Pulmonary and Mediastinal Pathology from the Department of Defence, Armed Forces Institute of Pathology, Washington DC, USA for his review and comment on this case.

1 Mark EJ. Mesenchymal cystic hamartoma of the lung. $N$ Engl f Med 1986;315:1255-9.

2 Willis RA. Pathology of tumours. St Louis: CV Mosby, 1948. 3 Graham JM Jr, Boyle W, Troxell J, Cullity GJ, Sprague PL, Beckwith JB. Cystic hamartoma of lung and kidney: a spectrum of developmental abnormalities. Am 7 Med Genet 1987;27:45-59.

4 Holden WE, Mulkey DD, Kessler S. Multiple peripheral lung cysts and hemoptysis in an otherwise asymptomatic adult. Am Rev Respir Dis 1982;126:930-2.

5 Stephanopoulos C, Catsaras H. Myxosarcoma complicating a cystic hamartoma of the lung. Thorax 1963;18:144-5.

6 Bove KE. Sarcoma arising in pulmonary mesenchymal cystic hamartoma. Pediatr Pathol 1989;9:785-92.

7 Hedlund GL, Bisset GS, Bove KE. Malignant neoplasms arising in cystic hamartomas of the lung in childhood. Radiology 1989;173:77-9.

\section{Department of} Cardiac Surgery, Bristol Royal Infirmary, Bristol BS2 8HW, UK A D Fox

Southmead Hospital Westbury-on-Trym,
Bristol BS10 5NB, UK S E Robbins

Reprint requests to: Mr A D Fox, 59 Elton Road, Bishopston, Bristol BS7 8DG, UK.

Received 30 September 1993

Returned to authors 24 November 1993 Revised version received 13 December 1993 Accepted for publication 6 January 1994

\section{Aortic valvulitis complicating Wegener's granulomatosis}

\author{
A D Fox, S E Robbins
}

\begin{abstract}
In a case of Wegener's granulomatosis aortic valve replacement was performed for worsening congestive cardiac failure secondary to aortic incompetence. Two paravalvular lesions and an isolated intraleaflet deficiency of the non-coronary cusp were identified at operation. Histological changes were consistent with a connective tissue disease.
\end{abstract}

(Thorax 1994;49:1176-1177)

\section{Case report}

A 20 year old man presented with arthralgia, haemoptysis, inflamed fauces, conjunctivitis and weight loss. On clinical examination he was distressed, pale, febrile $\left(39.3^{\circ} \mathrm{C}\right)$ with a tachycardia (blood pressure $110 / 80 \mathrm{~mm} \mathrm{Hg}$ ). A vasculitic rash was present on his shins and elbows. Auscultation revealed bibasilar fine crackles and a blowing left parasternal diastolic murmur. He was anaemic (haemoglobin 8.7 $\mathrm{g} / \mathrm{dl})$ with a leucocytosis $\left(13.2 \times 10^{9} / 1\right)$, urea $12.5 \mathrm{mmol} / \mathrm{l}$ and creatinine $199 \mu \mathrm{mol} / \mathrm{l}$. Radiographic examination demonstrated cardiomegaly and fluffy basilar pulmonary opacifications. Echocardiography showed mild aortic and mitral regurgitation, mild left ventricular dilatation, and normal valve cusps. No evidence of pulmonary hypertension or vegetations was demonstrated. Renal ultrasound revealed normal sized kidneys of increased echogenicity consistent with glomerulonephritis. Antineutrophil cytoplasmic antibody (ANCA) titres $(>1 / 320)$ confirmed the diagnosis. Treatment with prednisolone, cyclophosphamide and plasma exchange commenced immediately.

Five months later worsening congestive cardiac failure, recurrent epistaxes, pleurisy and anaemia (haemoglobin $5.9 \mathrm{~g} / \mathrm{dl}$ ) necessitated readmission. Echocardiography revealed left ventricular dilatation and partially prolapsing, thin aortic valve cusps. Despite treatment his condition deteriorated.

Examination demonstrated a collapsing pulse, blood pressure of $240 / 120 \mathrm{~mm} \mathrm{Hg}$, a displaced apex beat, a precordial thrill, and a pandiastolic murmur. Bibasilar crepitations persisted. A diagnosis of severe aortic incompetence with significant left ventricular failure was made.

Left axis deviation and left ventricular hypertrophy (without strain) was noted on the electrocardiogram. Repeat echocardiography confirmed a moderately dilated left ventricle with good contractility, very severe aortic regurgitation with prolapsing aortic cusps, a normal mitral valve, and an ejection fraction of 